\title{
Solitary-Solution Formulation for Differential-Difference Equations Using an Ancient Chinese Algorithm
}

\author{
Ji-Huan He, ${ }^{1}$ S. K. Elagan, ${ }^{2,3}$ and Guo-Cheng $\mathrm{Wu}^{4,5}$ \\ ${ }^{1}$ National Engineering Laboratory for Modern Silk, College of Textile and Engineering, Soochow University, \\ 199 Ren-ai Road, Suzhou 215123, China \\ ${ }^{2}$ Mathematics E Statistics Department, Faculty of Science, Taif University, P.O. Box 888, Saudi Arabia \\ ${ }^{3}$ Department of Mathematics, Faculty of Science, Menofiya University, Shebin Elkom, Egypt \\ ${ }^{4}$ Key Laboratory of Numerical Simulation of Sichuan Province, Neijiang, Sichuan 641112, China \\ ${ }^{5}$ College of Mathematics and Information Science, Neijiang Normal University, Neijiang, Sichuan 641112, \\ China
}

Correspondence should be addressed to Ji-Huan He, hejihuan@suda.edu.cn

Received 6 December 2011; Accepted 10 January 2012

Academic Editor: Allan C. Peterson

Copyright (C) 2012 Ji-Huan He et al. This is an open access article distributed under the Creative Commons Attribution License, which permits unrestricted use, distribution, and reproduction in any medium, provided the original work is properly cited.

This paper applies an ancient Chinese algorithm to differential-difference equations, and a solitarysolution formulation is obtained. The discrete $\mathrm{mKdV}$ lattice equation is used as an example to elucidate the solution procedure.

\section{Introduction}

Discrete nonlinear lattices have been the focus of considerable attention in various branches of science. Many differential equations on the nanoscales are invalid, but the problems arising can be well modeled by differential-difference equations [1-5]. As is well known, there are many physically interesting problems such as charge fluctuations in net work, ladder type, electric circuits, phenomena in crystals, and molecular chains, which all can be modelled by differential-difference equations [6-9].

Recently many analytical methods were proposed to solve differential-difference equations, such as the exp-function method [10-12], the variational iteration method [1315], the homotopy perturbation method [16-19], and the parameterized perturbation method [17]. A complete review on various analytical methods is available in [18, 19]. In this paper we will apply an ancient Chinese algorithm to the discussed problem. 


\section{Amplitude-Frequency Formulation for Nonlinear Oscillators}

The ancient Chinese algorithm was first applied to nonlinear oscillators in 2006 [18], where a simple frequency formulation is given. Consider a generalized nonlinear oscillator in the form

$$
u^{\prime \prime}+f(u)=0, \quad u(0)=A, \quad u^{\prime}(0)=0 .
$$

We use two trial functions

$$
\begin{gathered}
u_{1}(t)=A \cos \omega_{1} t \\
u_{2}=A \cos \omega_{2} t
\end{gathered}
$$

which are, respectively, the solutions of the following linear oscillator equations:

$$
\begin{aligned}
& u^{\prime \prime}+\omega_{1}^{2} u=0, \\
& u^{\prime \prime}+\omega_{2}^{2} u=0,
\end{aligned}
$$

where $\omega_{1}$ and $\omega_{2}$ are trial frequencies which can be chosen freely; for example, we can set $\omega_{1}=1$ and $\omega_{2}=2$ or $\omega_{1}=1$ and $\omega_{2}=\omega$, where $\omega$ is assumed to be the frequency of the nonlinear oscillator.

Substituting (2.2) and (2.3) into, respectively, (2.1), we obtain the following residuals:

$$
\begin{aligned}
& R_{1}(t)=-A \omega_{1}^{2} \cos \omega_{1} t+f\left(A \cos \omega_{1} t\right) \\
& R_{2}(t)=-A \omega_{2}^{2} \cos \omega_{2} t+f\left(A \cos \omega_{2} t\right)
\end{aligned}
$$

According to the ancient Chinese algorithm, an amplitude-frequency formulation for nonlinear oscillators was proposed [18].

$$
\omega^{2}=\frac{\omega_{1}^{2} R_{2}(0)-\omega_{2}^{2} R_{1}(0)}{R_{2}(0)-R_{1}(0)} .
$$

Other modifications of the frequency formulation are shown in [20-23]. The frequency formulation is now widely used to solve various nonlinear oscillators [20-25], and the basic idea of the Chinese algorithm can also be used to solve other nonlinear problems [26-29].

\section{Solitary-Solution Formulation for Differential-Difference Equations}

Suppose the differential-difference equation we discuss in this paper is in the following nonlinear polynomial form:

$$
\frac{d u_{n}(t)}{d t}=f\left(u_{n-1}, u_{n}, u_{n+1}\right),
$$

where $u_{n}=u(n, t)$ is a dependent variable, and $t$ is a continuous variable, and $n, p_{i} \in Z$. 
Using the basic idea of the ancient Chinese algorithm, we choose two trial functions in the following forms:

$$
\begin{aligned}
& u_{n, 1}(n, t)=f\left(\xi_{n}+\omega_{1} t\right), \\
& u_{n, 2}(n, t)=g\left(\xi_{n}+\omega_{2} t\right),
\end{aligned}
$$

where $\xi_{n}=n d+\xi_{0}, \quad \xi_{0}$ is arbitrary, and $f$ and $g$ are known functions. If a periodic solution is searched for, $f$ and $g$ must be periodic functions; if a solitary solution is solved, $f$ and $g$ must be of solitary structures. In this paper a bell solitary solution of a differential-difference equation is considered, and trial functions are chosen as follows:

$$
\begin{aligned}
& u_{n, 1}(n, t)=\frac{A}{e^{\xi_{n}+\omega_{1} t}+e^{-\left(\xi_{n}+\omega_{1} t\right)}+B}, \quad \omega_{1}=1, \\
& u_{n, 2}(n, t)=\frac{A}{e^{\xi_{n}+\omega t}+e^{-\left(\xi_{n}+\omega t\right)}+B}, \quad \omega_{2}=\omega .
\end{aligned}
$$

For $u_{n}, u_{n-1}$, and $u_{n+1}$ should be compatible; then, from (3.3), we have

$$
\begin{aligned}
& u_{n-1,1}(n, t)=\frac{A}{e^{\xi_{n}-d+t}+e^{-\left(\xi_{n}-d+t\right)}+B^{\prime}} \\
& u_{n+1,1}(n, t)=\frac{A}{e^{\xi_{n}+d+t}+e^{-\left(\xi_{n}+d+t\right)}+B^{\prime}}
\end{aligned}
$$

and from (3.4), we have

$$
\begin{aligned}
& u_{n-1,2}(n, t)=\frac{A}{e^{\xi_{n}-d+\omega t}+e^{-\left(\xi_{n}-d+\omega t\right)}+B}, \\
& u_{n+1,2}(n, t)=\frac{A}{e^{\xi_{n}+d+\omega t}+e^{-\left(\xi_{n}+d+\omega t\right)}+B} .
\end{aligned}
$$

Solution Procedure

Step 1. Define residual function

$$
\tilde{R}(t)=\frac{d u_{n}(t)}{d t}-f\left(u_{n-1}, u_{n}, u_{n+1}\right)
$$

Substituting (3.3)-(3.7) into (3.1), we can obtain, respectively, the residual functions $\widetilde{R}_{1}$ and $\widetilde{R}_{2}$

$$
\begin{aligned}
& \tilde{R}_{1}(t)=\frac{d u_{n, 1}(t)}{d t}-f\left(u_{n-1,1}, u_{n, 1}, u_{n+1,1}\right), \\
& \tilde{R}_{2}(t)=\frac{d u_{n, 2}(t)}{d t}-f\left(u_{n-1,2}, u_{n, 2}, u_{n+1,2}\right) .
\end{aligned}
$$


Step 2. Solitary-solution formulation is constructed as follows:

$$
\omega^{2}=\frac{\omega_{1}^{2} \widetilde{R}_{2}(0)-\omega_{2}^{2} \widetilde{R}_{1}(0)}{\widetilde{R}_{2}(0)-\widetilde{R}_{1}(0)}
$$

where $\omega_{1}=1$ and $\omega_{2}=\omega$.

Step 3. Combining the coefficients of $e^{\xi_{n}}$ in (3.10), and setting them to be zero, we can solve the algebraic equations to find the values of $\omega, A$, and $B$. Finally an explicit solution is obtained.

\section{Application in Discrete mKdV Lattice}

The famous mKdV lattice equation reads [10]

$$
\frac{d u_{n}}{d t}=\left(\alpha-u_{n}^{2}\right)\left(u_{n-1}-u_{n+1}\right)
$$

where $\alpha \neq 0$.

Substituting (3.3)-(3.7) into (4.1), we obtain, respectively, the residual functions $\widetilde{R}_{1}$ and $\widetilde{R}_{2}$, and using the solitary-solution formulation, (3.10), we have

$$
\begin{aligned}
& e^{3 \xi_{n}}\left(\omega^{3}+\omega^{2} \alpha e^{-d}-\omega^{2} \alpha e^{d}-\omega-\alpha e^{-d}+\alpha e^{d}\right)-e^{-3 \xi_{n}}\left(\omega^{3}+\omega^{2} \alpha e^{-d}-\omega^{2} \alpha e^{d}-\omega-\alpha e^{-d}+\alpha e^{d}\right) \\
& +e^{2 \xi_{n}} B\left(\omega^{3} e^{-d}+\omega^{3} e^{d}-2 \omega^{2} \alpha e^{d}+2 \omega^{2} \alpha e^{-d}-\omega e^{d}-\omega e^{-d}+2 \alpha e^{d}-2 \alpha e^{-d}\right) \\
& -e^{-2 \xi_{n}} B\left(\omega^{3} e^{-d}+\omega^{3} e^{d}-2 \omega^{2} \alpha e^{d}+2 \omega^{2} \alpha e^{-d}-\omega e^{d}-\omega e^{-d}+2 \alpha e^{d}-2 \alpha e^{-d}\right) \\
& +e^{\xi_{n}}\left(\omega^{3} B^{2}-\omega^{3}+\omega^{3} e^{-2 d}+\omega^{3} e^{2 d}+\omega^{2} A^{2} e^{d}-\omega^{2} A^{2} e^{-d}+\omega^{2} \alpha e^{-d}-\omega^{2} \alpha e^{d}+\omega^{2} \alpha B^{2} e^{-d}\right. \\
& \left.\quad-\omega^{2} \alpha B^{2} e^{d}-\omega B^{2}+\omega-\omega e^{2 d}-\omega e^{-2 d}+A^{2} e^{-d}-A^{2} e^{d}+\alpha B^{2} e^{d}-\alpha B^{2} e^{-d}+\alpha e^{d}-\alpha e^{-d}\right) \\
& -e^{-\xi_{n}}\left(\omega^{3} B^{2}-\omega^{3}+\omega^{3} e^{-2 d}+\omega^{3} e^{2 d}+\omega^{2} A^{2} e^{d}-\omega^{2} A^{2} e^{-d}+\omega^{2} \alpha e^{-d}-\omega^{2} \alpha e^{d}+\omega^{2} \alpha B^{2} e^{-d}\right. \\
& \left.\quad-\omega^{2} \alpha B^{2} e^{d}-\omega B^{2}+\omega-\omega e^{2 d}-\omega e^{-2 d}+A^{2} e^{-d}-A^{2} e^{d}+\alpha B^{2} e^{d}-\alpha B^{2} e^{-d}+\alpha e^{d}-\alpha e^{-d}\right) \\
& =0 .
\end{aligned}
$$

Setting the coefficients of $e^{i \xi_{n}}$ to be zero, we have

$$
\begin{aligned}
& \omega^{3}+\omega^{2} \alpha e^{-d}-\omega^{2} \alpha e^{d}-\omega-\alpha e^{-d}+\alpha e^{d}=0, \\
& B\left(\omega^{3} e^{-d}+\omega^{3} e^{d}-2 \omega^{2} \alpha e^{d}+2 \omega^{2} \alpha e^{-d}-\omega e^{d}-\omega e^{-d}+2 \alpha e^{d}-2 \alpha e^{-d}\right)=0 \\
& \omega^{3} B^{2}-\omega^{3}+\omega^{3} e^{-2 d}+\omega^{3} e^{2 d}+\omega^{2} A^{2} e^{d}-\omega^{2} A^{2} e^{-d}+\omega^{2} \alpha e^{-d}-\omega^{2} \alpha e^{d}+\omega^{2} \alpha B^{2} e^{-d}, \\
& \quad-\omega^{2} \alpha B^{2} e^{d}-\omega B^{2}+\omega-\omega e^{2 d}-\omega e^{-2 d}+A^{2} e^{-d}-A^{2} e^{d}+\alpha B^{2} e^{d}-\alpha B^{2} e^{-d}+\alpha e^{d}-\alpha e^{-d}=0 .
\end{aligned}
$$


Solving (4.3) simultaneously, we have

$$
\begin{gathered}
\omega=2 \alpha \sinh (d), \\
A=2 \sqrt{-\alpha} \sinh (d), \\
B=0 .
\end{gathered}
$$

We, therefore, obtain the following needed solitary solution:

$$
u_{n}=\frac{2 \sqrt{-\alpha} \sinh (d)}{e^{\xi_{n}+2 \alpha \sinh (d) t}+e^{-\left(\xi_{n}+2 \alpha \sinh (d) t\right)}}=\sqrt{-\alpha} \sinh (d) \operatorname{sech}\left\{2 \alpha \sinh (d) t+n d+\xi_{0}\right\} .
$$

\section{Conclusions}

Though there are many analytical methods, such as the exp-function method, the variational iteration method, and the homotopy perturbation method, for differential-difference equations, this paper suggests an effective and simple approach to such problems using the basic idea of the ancient mathematics, and the simple formulation can be used routinely by followers to various differential-difference equations.

\section{Acknowledgment}

The work is supported by a project funded by the Priority Academic Program Development of Jiangsu Higher Education Institutions (PAPD).

\section{References}

[1] G. C. Wu, L. Zhao, and J. H. He, "Differential-difference model for textile engineering," Chaos, Solitons and Fractals, vol. 42, no. 1, pp. 352-354, 2009.

[2] J.-H. He and S.-D. Zhu, "Differential-difference model for nanotechnology," Journal of Physics, vol. 96, no. 1, Article ID 012189, 2008.

[3] J. H. He, "An elementary introduction to recently developed asymptotic methods and nanomechanics in textile engineering," International Journal of Modern Physics B, vol. 22, no. 21, pp. 3487-3578, 2008.

[4] J. H. He, "Frontier of modern textile engineering and short remarks on some topics in physics," International Journal of Nonlinear Sciences and Numerical Simulation, vol. 11, no. 7, pp. 555-563, 2010.

[5] J. H. He and E. W. M. Lee, "Variational principle for the differential-difference system arising in stratified hydrostatic flows," Physics Letters Section A, vol. 373, no. 18-19, pp. 1644-1645, 2009.

[6] D. Baldwin, U. Goktas, and W. Hereman, "Symbolic computation of hyperbolic tangent solutions for nonlinear differential-difference equations," Computer Physics Communications, vol. 162, no. 3, pp. 203-217, 2004.

[7] M. J. Ablowtiz and J. F. Ladik, "On the solution of a class of nonlinear partial difference equations," Studies in Applied Mathematics, vol. 57, pp. 1-12, 1977.

[8] V. E. Adler, S. I. Svinolupov, and R. I. Yamilov, "Multi-component Volterra and Toda type integrable equations," Physics Letters Section A, vol. 254, no. 1-2, pp. 24-36, 1999.

[9] M. Toda, Theory of Nonlinear Lattices, Springer, Berlin, Germany, 1988.

[10] S.-D. Zhu, "Exp-function method for the discrete mKdV lattice," International Journal of Nonlinear Sciences and Numerical Simulation, vol. 8, no. 3, pp. 465-468, 2007.

[11] C. Q. Dai and Y. Y. Wang, "Exact travelling wave solutions of toda lattice equations obtained via the exp-function method," Zeitschrift fur Naturforschung Section A, vol. 63, no. 10-11, pp. 657-662, 2008.

[12] S. Zhang and H.-Q. Zhang, "Exp-function method for N-soliton solutions of nonlinear differentialdifference equations," Zeitschrift fur Naturforschung Section A, vol. 65, no. 11, pp. 924-934, 2010. 
[13] J. H. He, G. C. Wu, and F. Austin, "The variational iteration method which should be followed," Nonlinear Science Letters A, vol. 1, pp. 1-30, 2010.

[14] N. Faraz, Y. Khan, and F. Austin, "An alternative approach to differential-difference equations using the variational iteration method," Zeitschrift fur Naturforschung Section A, vol. 65, no. 12, pp. 10551059, 2010.

[15] A. Yildirim, "Applying he's variational iteration method for solving differential- difference equation," Mathematical Problems in Engineering, vol. 2008, Article ID 869614, 7 pages, 2008.

[16] M. M. Mousa, A. Kaltayev, and H. Bulut, "Extension of the homotopy perturbation method for solving nonlinear differential-difference equations," Zeitschrift fur Naturforschung Section A, vol. 65, no. 12, pp. 1060-1064, 2010.

[17] M. M. Mousa and A. Kaltayev, "Homotopy perturbation method for solving nonlinear differentialdifference equations," Zeitschrift fur Naturforschung Section A, vol. 65, no. 6-7, pp. 511-517, 2010.

[18] A. Yildirim, "Exact solutions of nonlinear differential-difference equations by he's homotopy perturbation method," International Journal of Nonlinear Sciences and Numerical Simulation, vol. 9, no. 2, pp. 111-114, 2008.

[19] A. Yildirim, "He's homotopy perturbation method for nonlinear differential-difference equations," International Journal of Computer Mathematics, vol. 87, no. 5, pp. 992-996, 2010.

[20] X. H. Ding and L. Zhang, "Applying He's parameterized perturbation method for solving differentialdifference equation," International Journal of Nonlinear Sciences and Numerical Simulation, vol. 10, no. 9, pp. 1249-1252, 2009.

[21] J. H. He, "Some asymptotic methods for strongly nonlinear equations," International Journal of Modern Physics B, vol. 20, no. 10, pp. 1141-1199, 2006.

[22] D. D. Ganji and S. H. H. Kachapi, "Analytical and numerical methods in engineering and applied sciences," Progress in Nonlinear Science, vol. 3, pp. 1-579, 2011.

[23] L. Geng and X. C. Cai, "He's frequency formulation for nonlinear oscillators," European Journal of Physics, vol. 28, no. 5, pp. 923-931, 2007.

[24] X. C. Cai and J. F. Liu, "Application of the modified frequency formulation to a nonlinear oscillator," Computers and Mathematics with Applications, vol. 61, no. 8, pp. 2237-2240, 2011.

[25] J. H. He, "Comment on "He"s frequency formulation for nonlinear oscillators'," European Journal of Physics, vol. 29, no. 4, pp. L19-L22, 2008.

[26] J. H. He, "An improved amplitude-frequency formulation for nonlinear oscillators," International Journal of Nonlinear Sciences and Numerical Simulation, vol. 9, no. 2, pp. 211-212, 2008.

[27] H. L. Zhang and L. J. Qin, "An ancient Chinese mathematical algorithm and its application to nonlinear oscillators," Computers and Mathematics with Applications, vol. 68, no. 1, pp. 2071-2075, 2011.

[28] A. E. Ebaid, "Oscillations in an $x(2 n+2) /(2 n+1)$ potential via he's frequency-amplitude formulation," Zeitschrift fur Naturforschung Section A, vol. 64, no. 12, pp. 877-878, 2009.

[29] J. H. He, "Application of ancient Chinese mathematics to optimal problems," Nonlinear Science Letters A, vol. 2, pp. 81-84, 2011. 


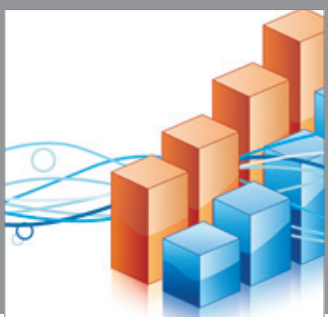

Advances in

Operations Research

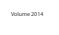

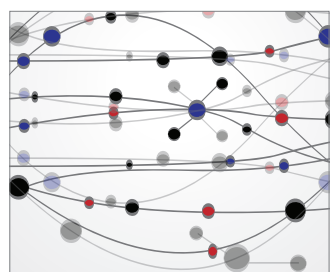

\section{The Scientific} World Journal
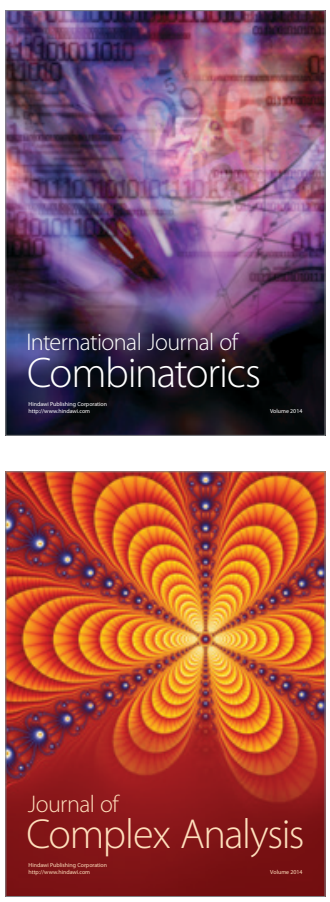

International Journal of

Mathematics and

Mathematical

Sciences
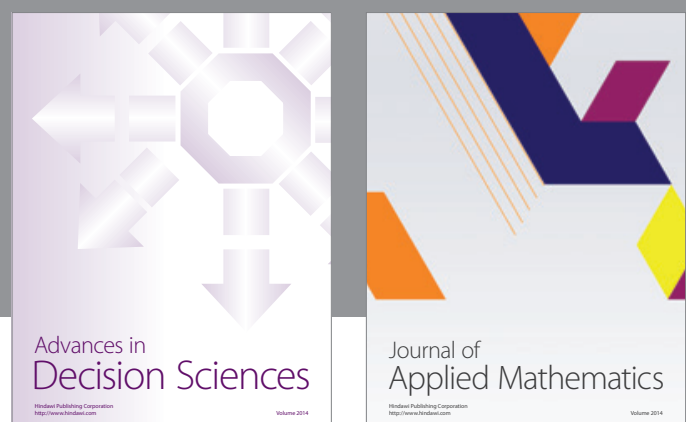

Journal of

Applied Mathematics
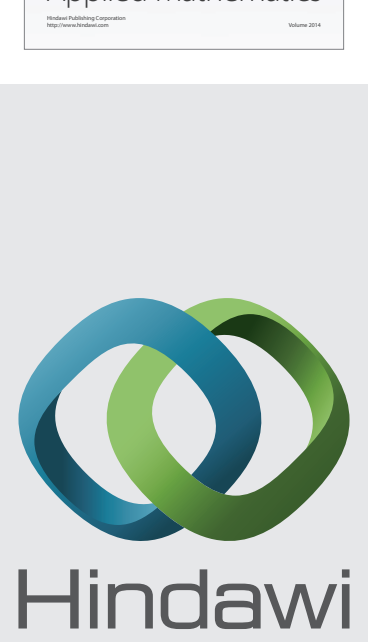

Submit your manuscripts at http://www.hindawi.com
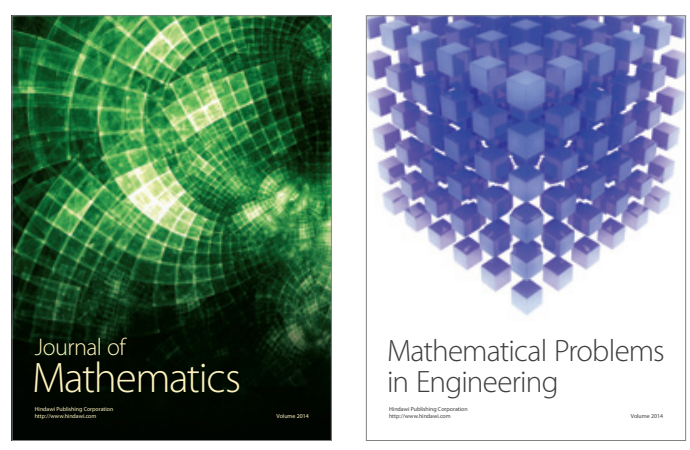

Mathematical Problems in Engineering
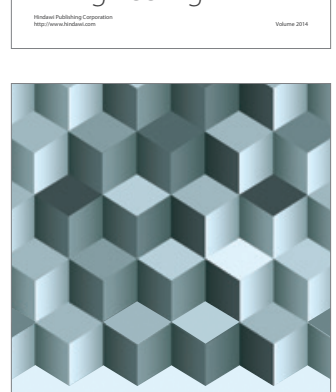

Journal of

Function Spaces
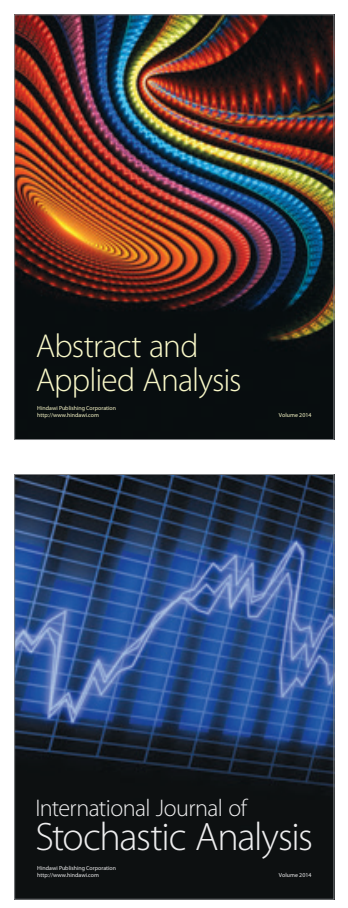

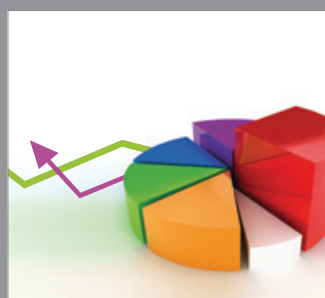

ournal of

Probability and Statistics

Promensencen
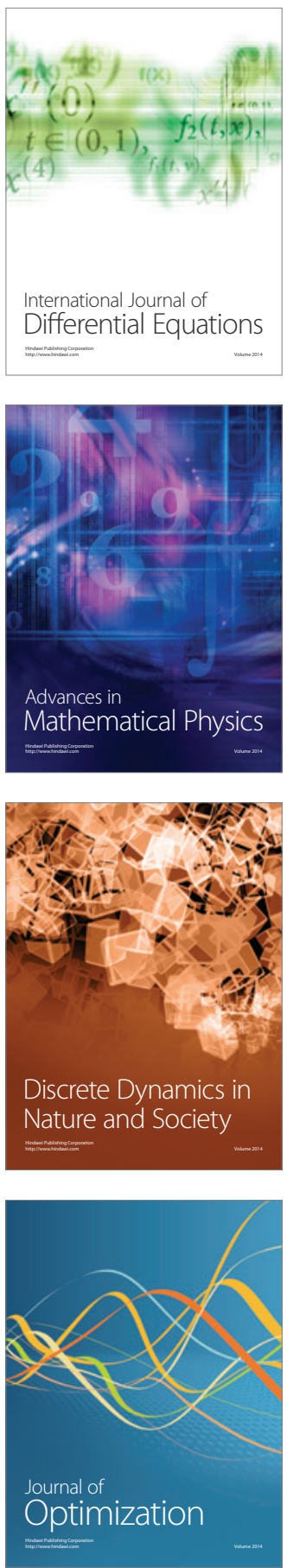